University of New Hampshire

University of New Hampshire Scholars' Repository

Space Science Center

Institute for the Study of Earth, Oceans, and

Space (EOS)

$2-1-2003$

\title{
SONTRAC: an imaging spectrometer for solar neutrons
}

James M. Ryan

University of New Hampshire, James.Ryan@unh.edu

L Desorgher

University of Berne

E O. Fluckiger

University of Bern

John R. Macri

University of New Hampshire - Main Campus, John.Macri@unh.edu

Mark L. McConnell

University of New Hampshire - Main Campus, mark.mcconnell@unh.edu

See next page for additional authors

Follow this and additional works at: https://scholars.unh.edu/ssc

Part of the Astrophysics and Astronomy Commons

\section{Recommended Citation}

James M. Ryan ; Laurent Desorgher ; Erwin O. Flueckiger ; John R. Macri ; Mark L. McConnell and Richard S. Miller "SONTRAC: an imaging spectrometer for solar neutrons", Proc. SPIE 4853, Innovative Telescopes and Instrumentation for Solar Astrophysics, 399 (February 1, 2003); doi:10.1117/12.460368;

http://dx.doi.org/10.1117/12.460368

This Conference Proceeding is brought to you for free and open access by the Institute for the Study of Earth, Oceans, and Space (EOS) at University of New Hampshire Scholars' Repository. It has been accepted for inclusion in Space Science Center by an authorized administrator of University of New Hampshire Scholars' Repository. For more information, please contact Scholarly.Communication@unh.edu. 


\section{Authors}

James M. Ryan, L Desorgher, E O. Fluckiger, John R. Macri, Mark L. McConnell, and R S. Miller 


\title{
SONTRAC: an Imaging Spectrometer for Solar Neutrons
}

\author{
James M. Ryan ${ }^{\mathrm{a}}$, Laurent Desorgher ${ }^{\mathrm{b}}$, Erwin O. Flückiger ${ }^{\mathrm{b}}$, John R. Macri ${ }^{\mathrm{a}}$, Mark L. McConnell ${ }^{\mathrm{a}}$, \\ Richard S. Miller ${ }^{\mathrm{a}}$ \\ ${ }^{a}$ University of New Hampshire, ${ }^{b}$ Physikalisches Institut, Bern
}

\begin{abstract}
An instrument capable of unambiguously determining the energy and direction of incident neutrons has important applications in solar physics-as well as environmental monitoring and medical/radiological sciences. The SONTRAC (SOlar Neutron TRACking) instrument is designed to operate in the neutron energy range of 20-250 MeV. The measurement principle is based on non-relativistic double scatter of neutrons off ambient protons (n-p scattering) within a block of densely packed scintillating fibers. Using this double-scatter mode it is possible to uniquely determine neutron energy and direction on an event-by-event basis. A fully operational science model of such an instrument has been built using $300 \mu \mathrm{m}(250 \mu \mathrm{m}$ active $)$ scintillating fibers. The science model consists of a $5 \times 5 \times 5 \mathrm{~cm}$ cube of orthogonal plastic scintillating fiber layers. Two orthogonal imaging chains, employing image intensifiers and CCD cameras, allow full 3-dimensional reconstruction of scattered proton particle tracks. We report the results of the science model instrument calibration using $35-65 \mathrm{MeV}$ protons. The proton calibration is the first step toward understanding the instrument response to n-p scatter events. Preliminary results give proton energy resolution of $2 \%(6 \%)$ at 67.5 (35) $\mathrm{MeV}$, and angular resolution of $2^{\circ}\left(4.5^{\circ}\right)$ at 67.5 (35) $\mathrm{MeV}$. These measurements are being used to validate detailed instrument simulations that will be used to optimize the instrument design and develop quantitative estimates of science return. Based on the proton calibration, neutron energy and angular resolution for a $10 \times 10 \times 10 \mathrm{~cm}$ version of SONTRAC is expected to be $\sim 5 \%$ and $<10^{\circ}$, respectively, while the efficiency of the detector to double $n-p$ scatter events is approximately $1 \%$. We will also discuss ongoing opto-electronic developmental efforts and concepts for extending the instrument response to lower energies.
\end{abstract}

Keywords: Sun, neutrons, particle detection, high-energy imaging

\section{INTRODUCTION}

\subsection{Proton Acceleration Problem.}

Understanding the particle acceleration (both electrons and ions) to high energies is an important step in solving the solar flare problem. Although substantial observational and theoretical progress has been made during the last two solar cycles, gaining a complete understanding of the acceleration and transport mechanisms for protons and/or ions remains one of the key goals of solar physics research ${ }^{1-3}$. Some of the nagging problems are: (1) the rapid acceleration of highenergy electrons and protons (up to several hundred $\mathrm{MeV}$ ) on time scales of $\sim 1 \mathrm{~s}$; (2) GeV proton acceleration on time scales of $<1$ minute, (3) the acceleration of $\mathrm{GeV}$ protons for periods of at least $\sim 10$ hours, (4) the nature of the delay of the $\gamma$-ray line emission with respect to the emission in hard X-rays and (5) how the ions can possess a major fraction of the flare energy during the impulsive phase. To address these questions a better knowledge of the accelerated proton spectrum is necessary. (Here we use the term proton spectrum to refer both to the proton spectrum and to the ion spectrum.)

Nuclear $\gamma$-rays, neutrons and $\gamma$-rays associated with pions each provide important information about different parts of the proton spectrum at the flare site ${ }^{4}$. Nuclear $\gamma$-rays from the decay of excited carbon, oxygen and other heavier nuclei primarily originate from the part of the proton spectrum not far above the $\gamma$-ray production threshold, typically around 20 $\mathrm{MeV}$. The nuclear $\gamma$-ray production is heavily weighted toward the low end of the spectrum due to the declining number

Correspondence: E-mail: James.Ryan@unh.edu telephone: 603-862-3510; fax: 603-862-4685 
of protons at higher energies and the fact that the $\gamma$-ray production cross sections normally peak between 5 and $10 \mathrm{MeV}$. The absolute threshold for production of $\pi$ mesons from p-p collisions is around $300 \mathrm{MeV}$ (with the effective threshold being somewhat higher) but is closer to $200 \mathrm{MeV}$ for protons incident on heavier nuclei such as carbon. This means that information about the proton spectrum above $250 \mathrm{MeV}$ can be derived from the emission of $\gamma$ rays associated with pion production $^{5}$. Figure 1 illustrates how different particle and photon species measurements are necessary to sample or measure the full energetic proton population.

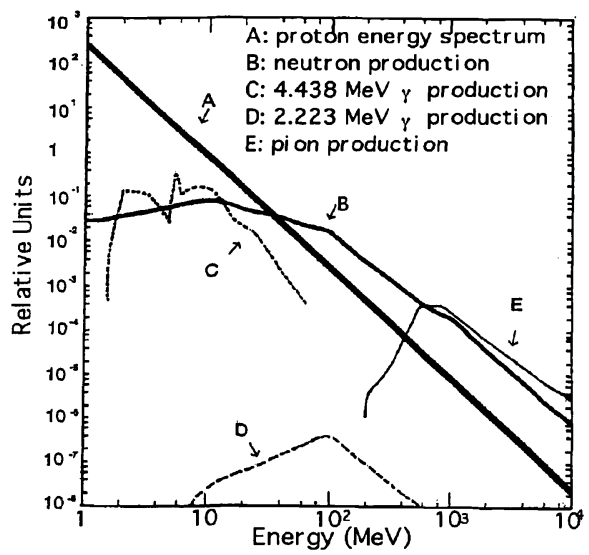

Fig. 1. Schematic representation of solar neutron and gamma-ray production.
The general shape of the spectrum in the range of $20-40 \mathrm{MeV}$ can be determined by $\gamma$-ray line ratios. This is a prime objective of the recently launched RHESSI mission, since it measures the $\gamma$-ray lines with unprecedented precision. The lines produced when protons strike the solar atmosphere are typically sharp and most have been unresolvable in earlier instruments because those instruments used scintillators. The line intensities, however, depend not only on the shape of the proton spectrum but also on the composition of the solar atmosphere, which shows signs of a depth dependence ${ }^{6}$. Conversely, when heavy ions strike the solar atmosphere, they produce Doppler broadened lines. The composition of the accelerated heavies must then be determined from the relative intensity of these broad lines $(\Delta E / E \sim 20 \%)$, a much more difficult task, because the lines overlap and meld with the bremsstrahlung continuum. Estimates of the composition of the accelerated ions are therefore subject to much greater uncertainties than similar estimates of the composition of the target material. However, through the measurement of sharp lines, RHESSI should afford great leaps in our understanding of the atmosphere's composition and the proton spectrum in the range of $10-40 \mathrm{MeV}$.

The spectrum above $250 \mathrm{MeV}$ must be deduced from the relative intensities of the $\pi^{0}$ emission $(69 \mathrm{MeV})$ versus the $\pi^{ \pm}$ secondary bremsstrahlung emission and the width of the $\pi^{0}$ feature. Here again, the secondary bremsstrahlung emission must be separated from any primary bremsstrahlung emission that is present below $70 \mathrm{MeV}$.

With the spectrum determined at the low end by way of $\gamma$-ray line ratios and determined at the high end by way of $\pi$ related emission one must question the need to measure anything else. Over the last two solar cycles we have witnessed a range of energetic flares, some exhibiting $\gamma$-ray emission above $50 \mathrm{MeV}$, but most not. Those that do not exhibit such emission yield no direct information on the proton spectrum above $30 \mathrm{MeV}$. For these events, we must rely on neutrons to provide that information. Neutrons are produced over a wide range of energies and retain a significant fraction of the incident proton energy. In the past we have sampled these neutrons indirectly by way of the $2.223 \mathrm{MeV} \gamma$-ray line emission. Free neutrons thermalize quickly in the solar atmosphere. Once thermalized, one of three things occurs: they undergo $\beta$ decay, they are captured on hydrogen producing a $2.223 \mathrm{MeV}$ line or they are captured on ${ }^{3} \mathrm{He}$ with no signal to interpret. In some cases, using the intensity of the $2.223 \mathrm{MeV}$ line relative to the intensity of other lines can provide information about the proton spectrum above $50 \mathrm{MeV}$. However, the $2.223 \mathrm{MeV}$ line is an awkward diagnostic. It first must be integrated over long periods of time because the capture times on ${ }^{3} \mathrm{He}$ and $\mathrm{H}$ are long (typically $\sim 100 \mathrm{~s}$ ), thereby integrating away spectral evolution information. Secondly, there are cases where using the $2.223 \mathrm{MeV}$ line can yield erroneous proton spectra, because neutron production takes place over such a wide energy range from all constituents of the solar atmosphere. Consequently, the $2.223 \mathrm{MeV}$ data do a relatively poor job of constraining the hardness of the underlying proton spectrum. The uncertainty of spectral hardness, the loss of temporal information and the potential variability of the ${ }^{3} \mathrm{He}$ abundance limit the utility of the $2.223 \mathrm{MeV}$ line as an independent measure of the proton/ion spectrum between 50 and $300 \mathrm{MeV}$. 
A better means of measuring the $50-300 \mathrm{MeV}$ proton spectrum is via direct measurement of neutrons in the same range. For a small number of events, this was done with the COMPTEL, OSSE and EGRET instruments on the Compton Observatory. Most relevant to this discussion are the COMPTEL measurements since they covered a similar range (with a similar technique) of neutron energy that our development instrument, SONTRAC, would.

One example is the 9 June 1991 solar flare. In the 9 June 1991 flare $^{7} \gamma$-ray lines, neutrons and $\pi$-related $\gamma$-ray emission were detected (Fig. 2). Inspection of the light curves of the three forms of emission show the following: the impulsive phase, rich in $\gamma$-ray lines, peaked in intensity at 0139 UT, whereas the neutron emission likely peaked later between 0140 and 0145 UT (velocity dispersion removed). The $20-200 \mathrm{MeV} \gamma$-ray signal peaked both during the impulsive phase and $\sim 0151 \mathrm{UT}^{8}$. (The 20-200 MeV signal was not deconvolved into components, so it likely contains bremsstrahlung and $\pi$ related components.) With the limited statistics, this fits a picture where during the impulsive phase we had an intense but relatively "normal" proton spectrum and an electron spectrum that produced $\gamma$ rays in the line region and above 20 $\mathrm{MeV}$. After the impulsive phase the proton spectrum evolved by dropping in intensity (line emission declined rapidly) and progressively hardening. In sequence, the line emission (20-50 MeV protons) peaked, followed by the neutron emission (50-300 MeV) and finally the pion production ( $>300 \mathrm{MeV})$, typical of other so-called long duration $\gamma$-ray flares 9 .

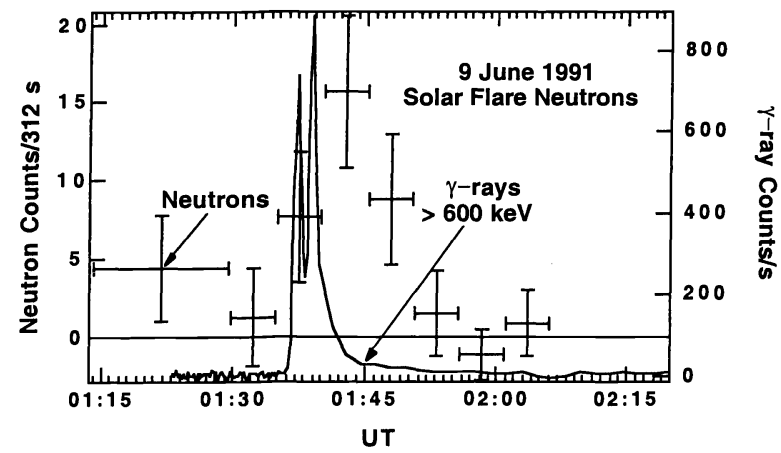

Fig. 2. Neutrons and $\gamma$-ray emission-time profiles for the 9 June 1991 solar flare, plotted at the time corresponding to a photon arrival time.

\subsection{Measuring Neutrons}

Bulk scintillators are efficient detectors of neutrons. Although they can be fashioned into large area instruments (such as SMM-GRS, OSSE and EGRETTASC), such instruments usually provide limited information about the energy of individual neutrons. Determining the neutron energy is important because the neutron production time at the Sun is determined from the neutron energy (velocity), the measured arrival time and the distance to the Sun. Without the neutron energy information, one must assume that the neutron production follows that of the (observable) $\gamma$-ray emission. However, this is an ad hoc assumption that typically does not reflect reality. If the energy of each neutron is measured accurately, the distribution's velocity dispersion can be removed and each neutron assigned to a unique origination time at the Sun. Then one only needs to model the spectral evolution of the neutron production to arrive at a proton spectrum. Also by using only fully measured neutrons, we simplify the instrument response. For a given energy, the response is simply described by an effective area rather than a convolution of interaction probabilities over all energies, making the results more robust. Our development instrument, SONTRAC, like COMPTEL, is capable of accurately measuring individual neutron energies and directions from the kinematics of the neutron detection physics without the use of neutron shields (see below).

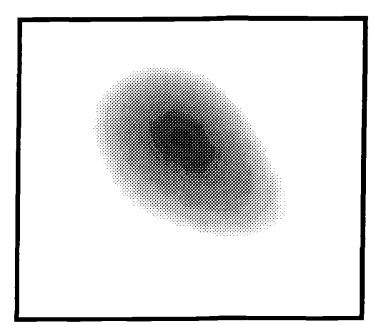

Fig. 3. COMPTEL image of the Sun in 10-80 MeV neutrons after the 15 June 1991 solar flare.
In space, neutron background is ubiquitous and intense. It is produced in the Earth's atmosphere and in the spacecraft itself. It is a problem for balloon platforms, low earth-orbiting spacecraft and interplanetary spacecraft. Anywhere there are interacting cosmic rays there will be neutrons. These background neutrons generally arrive isotropically, more or less, from the background emitting material that is typically distributed over a large solid angle as seen from the perspective of the instrument. The neutrons that COMPTEL measured from the June 1991 flares were only visible because of background rejection techniques used in double-scatter instruments. Simply put, the imaging properties of COMPTEL greatly reduced the acceptance solid angle for neutrons. The small solid angle that included the direction of the Sun constituted a small fraction of $4 \pi \mathrm{sr}$. It was neutrons from these directions that were analyzed for both events 
and to produce the neutron image of the Sun (Figure 3). One, in principle, could shield a bulk detector, but the shield must be massive since neutrons are so penetrating. If not thick enough, the shields will amplify the background rather than reduce it. Shields are not an efficient use of the mass budget of a payload.

\section{THE SONTRAC CONCEPT}

\subsection{The Detection Method.}

The details of the SONTRAC concept and prototype design are described below. However, it is safe to say that SONTRAC, by measuring more parameters associated with each neutron, can determine the incident direction and energy of a neutron. By comparison, COMPTEL only measured pulse heights, interaction locations and time of flight. This constrained the incident neutron directions to an annulus on the celestial sphere. If we can reduce our acceptance solid angle from an annulus to a small disk we will realize another large reduction in background. The new parameters that SONTRAC measures are the 3-d ionization tracks of the recoil protons from the incident neutron. By measuring the recoil tracks, we can collapse the annulus to a point (small disk). COMPTEL was an enormous and heavy instrument. SONTRAC will be small and light, but still providing similar effective area.

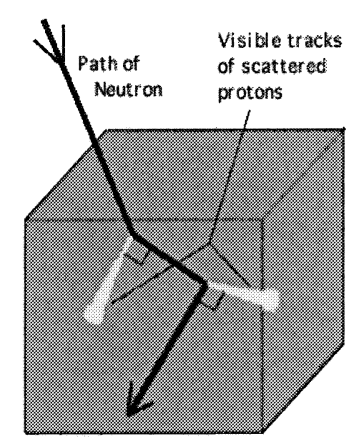

Fig. 4. Double scatter neutron event in a block of plastic scintillator.

The double-scatter of a non-relativistic neutron in a solid block of plastic scintillator is illustrated in Figure 4. Neutrons predominantly interact in plastic scintillator either by elastically scattering from hydrogen $(n-p)$ or by interacting with carbon (n-C). The n-p events are the most useful. For nonrelativistic scattering

$$
\sin ^{2} \phi_{n^{\prime}}=\cos ^{2} \phi_{p^{\prime}}=\frac{E_{p^{\prime}}}{E_{n^{\prime}}+E_{p^{\prime}}}=\frac{E_{p^{\prime}}}{E_{n}}
$$

where, $E_{n}$ is the incident neutron energy; $E_{n}$, and $E_{p^{\prime}}$, are the scattered neutron and proton energies, respectively; $\phi_{n}$, and $\phi_{p}$, are the neutron and proton scatter angles, respectively. The kinematics of nonrelativistic scattering implies that the scattered neutron and proton momenta lie at right angles to one another. At the highest energies for SONTRAC, these expressions are modified to account for relativistic effects.

If the incident direction of a given neutron is known, then the measurement of the energy and direction of a recoil proton in a single scattering is sufficient to determine the incident neutron energy. In particular, if the incident direction is known, then $E_{n}$, is determined and the neutron energy is,

$$
E_{n}=\frac{E_{p^{\prime}}}{\cos ^{2} \phi_{p^{\prime}}}
$$

Single n-p scatters do not allow determination of both neutron incident angle and energy simultaneously. A more deterministic approach is provided by double-scatter events. If both the energy and direction of the recoil protons in a double scatter event are measured, then the energy and incident direction of the neutron are uniquely determined. A system that can measure the parameters of both recoil proton tracks in three dimensions therefore provides the necessary information to unambiguously determine the incident neutron energy and direction. The instrumental angular and energy resolutions depend on the ability to precisely measure the energy and direction of the recoil protons. In the case of solar neutrons this capability helps in separating the spatially resolved source signal from background.

\subsection{Scintillating-Fiber Detector}

Rather than using a monolithic block of plastic scintillator with no spatial information, we use orthogonal plastic scintillating fibers. These fibers, arranged into a detector bundle, can measure incident neutrons in the 20 to $250 \mathrm{MeV}$ range by recording the image of the recoil proton tracks. The scintillating fibers serve both as the scattering centers and 
as light pipes for image readout. A fiber pitch of $300 \mu \mathrm{m}(250 \mu \mathrm{m}$ active fiber size) was selected so that a $10 \mathrm{MeV}$ proton traverses several fibers before stopping. Figure 5 represents a $3 \mathrm{~mm}$ cubic piece of such a bundle. The square fibers are arranged in stacked planes with the fibers in each plane orthogonal to those in the planes above and below. This alternating orientation of fiber planes provides a stereoscopic view of recoil proton tracks and allows the reconstruction of these tracks in three dimensions. These ionizing tracks in turn provide the information necessary to determine the arrival direction and energy of incident neutrons. The resulting neutron angular and energy resolutions depend upon the precision with which the recoil proton direction and energy can be determined.

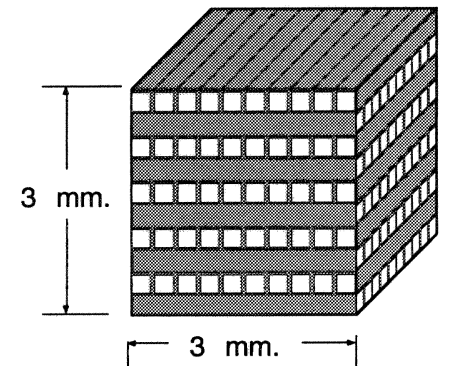

Fig. 5. Cutaway view: orthogonal scintillating fiber layers

The fibers are coupled to imaging electronics (currently image-intensified CCD cameras), one for each set of orthogonal fiber planes. The intensity of each pixel's recorded scintillation light combined with the inherent pixelization of the fiber bundle provides the information for track reconstruction. The ionization track length provides a good measure of the recoil proton energy. The Bragg peak, corresponding to the greater ionization near the end of a proton track, identifies the particle direction.

\subsection{The SONTRAC Science Model (SM)}

Figure 6 is an image of a double n-p scatter event recorded with our 2-d SONTRAC prototype. After successfully demonstrating the basic detection concept-self-triggered, stereoscopic imaging of charged particle tracks-with this prototype $e^{10-13}$, we constructed a true 3-d Science Model (SM) of the SONTRAC instrument (Figure 7). The SM consists of a $5 \times 5 \times 5 \mathrm{~cm}$ scintillating plastic fiber bundle with appropriately sized optoelectronic readout components. Fiber pitch is $300 \mu \mathrm{m}(250 \mu \mathrm{m}$ active material) in $x, y$ and $z$. The plastic scintillating fiber detector bundle (center), light guides, fiber optic tapers, image intensifiers, and associated power supplies are contained within a single light-tight enclosure (cover removed in photo). The two CCD cameras are mounted externally. The SM has no ancillary detectors such as anti-coincidence or calorimeter.

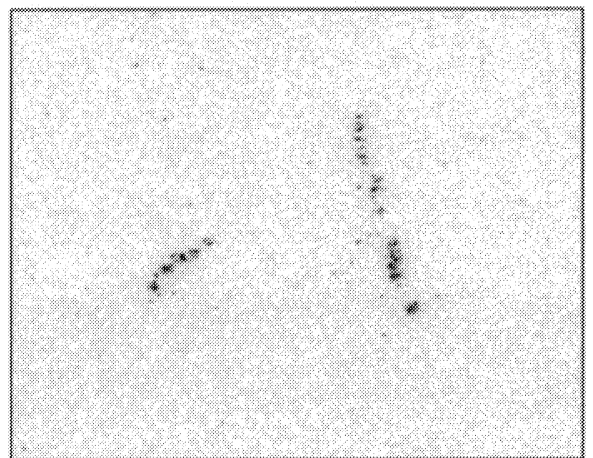

Fig. 6. A double n-p scatter from a 65 $\mathrm{MeV}$ neutron.

Coincident signals from the PMTs provide the trigger for gating the image intensifiers and capturing the two orthogonal track images with the CCD cameras. The starting location of the track and intensity along the track identify the type of radiation detected (e.g., proton, neutron, muon). For particles that range out within the fiber bundle, the ionization track length provides a good measure of the particle energy. The Bragg peak, corresponding to the greater ionization near the end of a particle track, identifies the particle direction. Protons that result from n-p scattering can be identified as originating within the fiber bundle. Charged particles that originate outside the bundle can be identified with tracks that begin at the edge of the bundle.

\subsection{Test and Calibration Results}

The signal measured at the image intensifier photocathode for minimumionizing cosmic-ray muon tracks is ample ( 4 photoelectrons per MIP in a single $250 \mu \mathrm{m}$ fiber). Highly ionizing recoil protons, however, are the primary probes used for detecting and measuring incident neutrons. As such, the ability to reconstruct these charged particle tracks is critical. Track direction, orientation, and length are the important parameters for determining the proton energy and scatter direction in three dimensions. Quantifying variations in reconstruction parameters as a function of proton energy is necessary to properly understand the instrument response.

A proton calibration of the SONTRAC SM was performed at the Crocker Nuclear Laboratory (CNL) (U.C. Davis) cyclotron in June 2000 and February 2001. The primary goal was to measure the instrument energy and angular resolutions using incident protons at several energies. Over 5000 proton events, all at normal incidence, were acquired at four different incident energies $(35,46.5,55,67.5 \mathrm{MeV})$. Figure 8 shows the angular response to $55 \mathrm{MeV}$ neutrons. 
Table 1 summarizes the proton track reconstruction performance based on these data. Other smaller data sets from incident neutrons covering the energy range of about 10 to $67.5 \mathrm{MeV}$ were also acquired during instrument calibration at CNL.

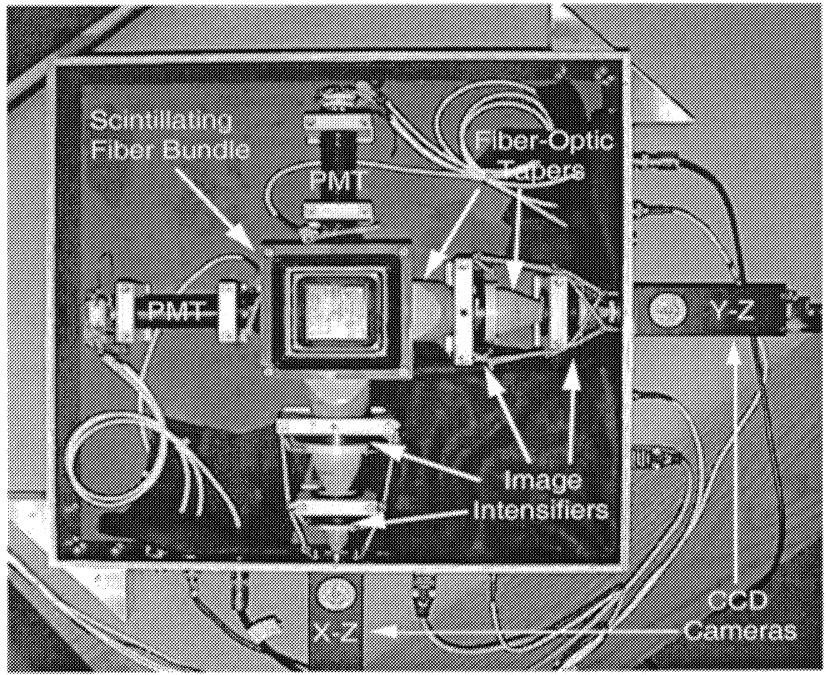

Fig. 7. SONTRAC Science Model assembly.

Our calibration data demonstrate the ability of SONTRAC to identify and discriminate minimumionizing cosmic-ray muons from highly ionizing particles such as incident or recoil protons. Typical CCD images of these events are shown in Figure 9. The box indicates the perimeter of the SM fiber bundle. Particles are incident from above. Minimum-ionizing muons are characterized by a uniform ionization intensity along their tracks, while proton tracks show greater density (from increased ionization losses) at the track endpoint (Bragg peak). Neutron events are easy to identify because they produce ionization tracks originating from within the fiber bundle while incident protons produce tracks that start at the surface of the detector. This particle identification capability is a unique and important feature for characterizing the radiation environment.

\subsection{Monte Carlo Simulations}

We developed a first version of a Monte Carlo model of the SONTRAC instrument ${ }^{14}$. Based on the GEANT4 library available from CERN, it includes models of the relevant proton-ionization energy losses, neutron-proton elastic scattering cross sections, etc., that are critical for accurate reproduction of the scintillating fiber bundle response to incident protons and neutrons. In the energy range covered by SONTRAC the GEANT4 standard model of neutron-

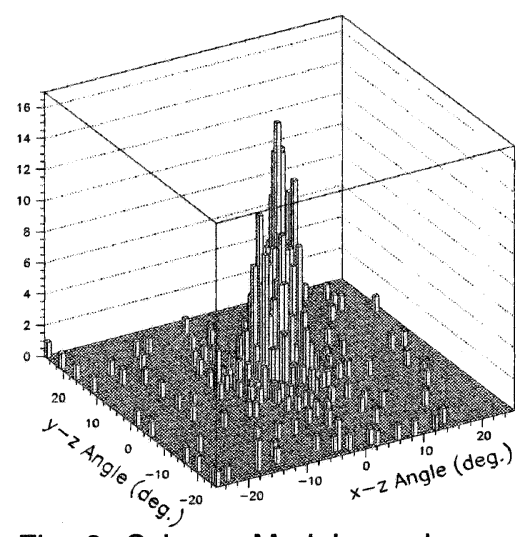

Fig. 8. Science Model angular response to $55 \mathrm{MeV}$ proton beam.

\begin{tabular}{|c|c|c|}
\hline \multicolumn{3}{|c|}{ Table 1} \\
\hline \multicolumn{3}{|c|}{ SM Proton Track Reconstruction } \\
\hline $\begin{array}{c}\mathbf{E}_{\mathrm{p}} \\
(\mathbf{M e V})\end{array}$ & $\left(\sigma_{E} / E\right)$ & $\begin{array}{c}\text { Ang. Res. } \\
(1 \sigma)\end{array}$ \\
\hline 35 & $4.8 \%$ & $4.6^{\circ}$ \\
\hline 46.5 & $3.4 \%$ & $4.0^{\circ}$ \\
\hline 55 & $2.8 \%$ & $3.2^{\circ}$ \\
\hline 67.5 & $2.1 \%$ & $2.3^{\circ}$ \\
\hline
\end{tabular}
proton and neutron carbon elastic scattering is not valid. For this reason an elastic model based on the ENDF database was developed and successfully tested.

The scintillating fiber bundle is modeled as a scintillating block with the same density as for the SONTRAC SM fiber core. Inside the scintillating block, the range and the direction of interacting protons are deduced from the start and stop positions of the proton tracks computed by the code. In Figure 10 the simulated mean proton range (solid line) is plotted versus incident energy and compared to proton calibration measurements of the SONTRAC SM (diamonds). The measured ranges are systematically lower than the simulation results. In the SONTRAC SM a $1 \mathrm{~mm}$ aluminum layer covers the top side of the detector. Proton energy loss in this plate reduces the length of a proton track. The dashed line in Figure 10 represents the range calculated by considering this aluminum layer in the GEANT4 simulation. Additional materials used at the Crocker Nuclear facility for reducing the proton beam intensity have been also included in the simulation, but their presence is not significant. The polystyrene core of the scintillating fiber is surrounded by two cladding layers and an extra coat of paint. By modeling the block as a mixture of polystyrene plus the material of the external layers we were still unable to account for the differences between the measured and predicted ranges. This discrepancy is still being investigated. 
In the neutron detection mode of the SONTRAC numerical model, range and directions of single and double recoil protons tracks are recorded. Although some of them come from n-C inelastic scattering, all recoil protons are interpreted as coming from single and double n-p elastic scatters. The proton energies are deduced from their ranges as already
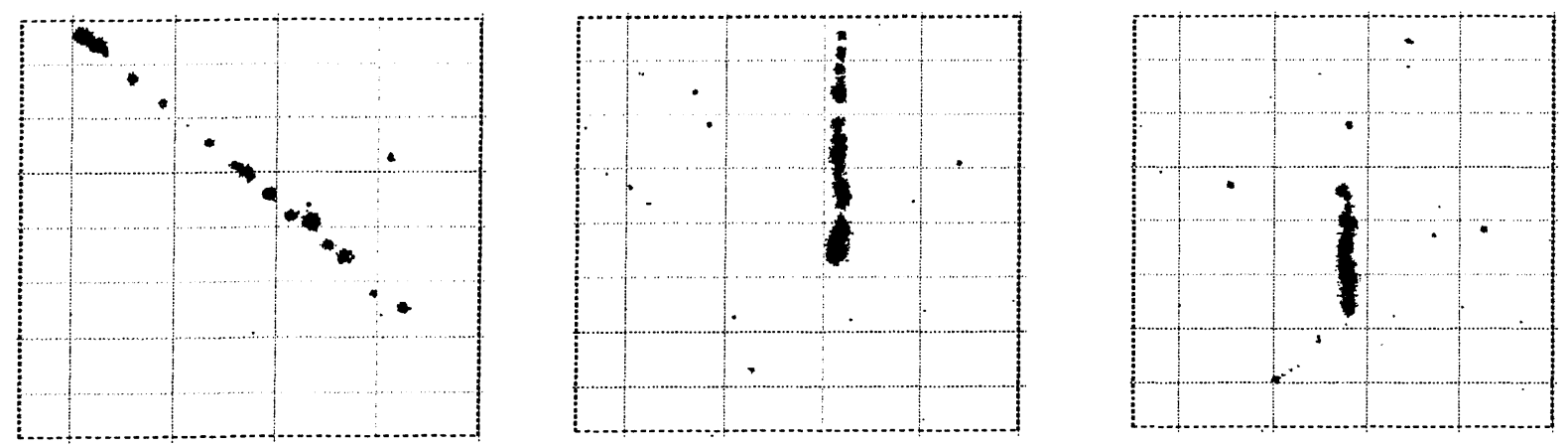

Fig. 9. Science Model track images illustrating response to different incident particle types: muon (left), incident proton (center), recoil proton from incident neutron (right).

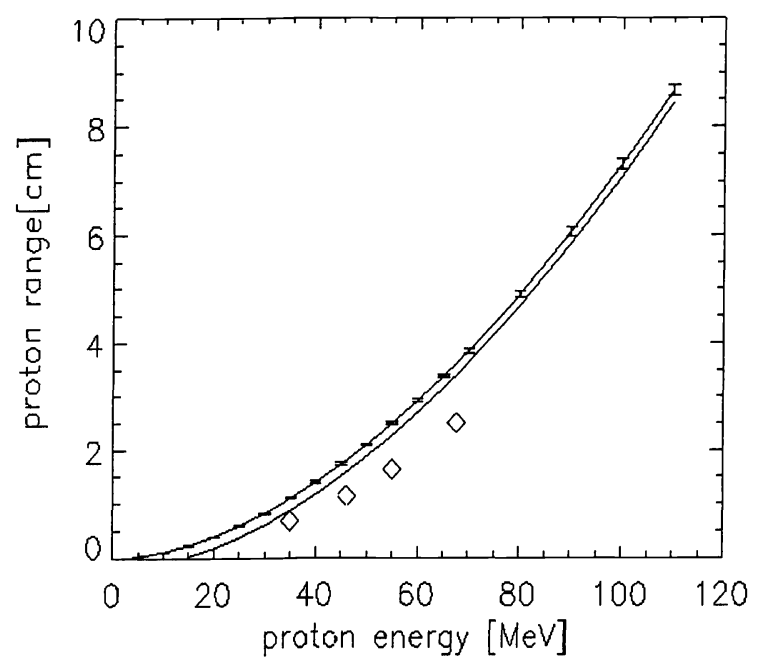

Fig. 10. Comparison of computed proton range and calibration measurements. explained. For single elastic scatters, the energy of an incident neutron is computed from the recoil-proton energy and an assumed incident direction. For double elastic scatters, the energy and direction of an incident neutron is computed from the energy and direction of both recoil protons. In Figure 11 we present the computed neutron energies in the single and double elastic scattering mode obtained for the interaction of 500,000 normal incident neutrons with energies of 50 $\mathrm{MeV}$ (shaded) and $100 \mathrm{MeV}$ (line). This simulation was done for a $(10 \mathrm{~cm})^{3}$ block. For both modes the peaks occur at the expected energies. In the single scattering mode the low energy part is contaminated by $\mathrm{n}-\mathrm{C}$ inelastic scatters, producing a background much like a Compton tail in a gamma-ray spectrometer. The contribution at higher energy is probably due to the poor direction resolution for low energy recoil protons with scattering directions close to $90^{\circ}$. For double scatters the

$\mathrm{n}-\mathrm{C}$ inelastic contamination at low energy is significantly reduced.

Using the proton calibration data to validate and fine-tune the model, it is possible to estimate the neutron response using a preliminary version of an instrument Monte Carlo calculation. For double n-p scatters the energy resolution is predicted from the calculations to be $3 \%$ at 50 and $100 \mathrm{MeV}$. The simulations predict neutron angular resolutions of better than $5-10^{\circ}$. The validation of the Monte Carlo code by calibration measurements is an important step in the SONTRAC development plan.

\subsection{Detection Capabilities of a Flight Version of SONTRAC}

Based on the instrument model we computed the response to a solar flare. We assumed the instrument to be a $(10 \mathrm{~cm})^{3}$ scintillator block located on a spacecraft at distances of 0.3 and $1 \mathrm{AU}$ from the Sun. For the assumed neutron emission we chose that of the 15 June 1991 solar flare ${ }^{15}$, i.e., $8.8 \times 10^{27} \mathrm{sr}^{-1}$ over 30 minutes (at $1 \mathrm{AU}$ ) in the energy range of 10-100 $\mathrm{MeV}$. At $1 \mathrm{AU}$, the neutron decay in flight effectively limits the range of the instrument to above $20 \mathrm{MeV}$. However, at $0.3 \mathrm{AU}, 10 \mathrm{MeV}$ neutrons suffer few decay losses, making the response of SONTRAC at $10 \mathrm{MeV}$ important. 


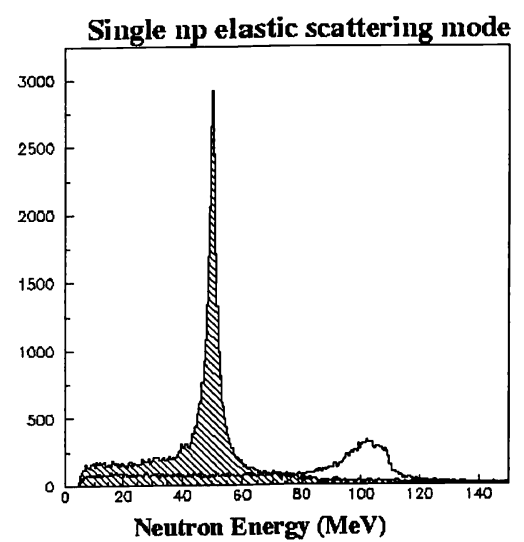

Double np elastic scattering mode

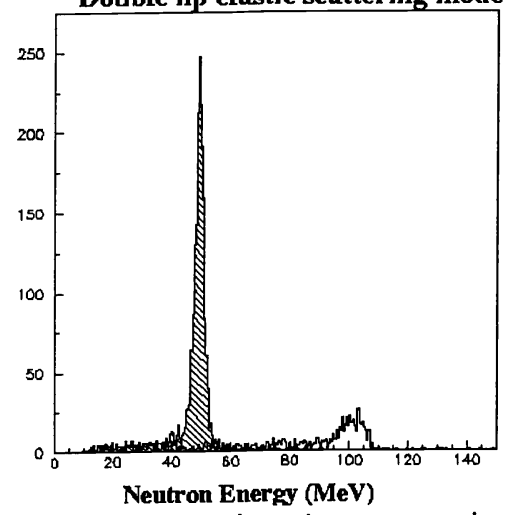

Fig. 11: Computed neutron energy in the single and double elastic scattering modes.
In Fig. 12 we show the 15 June 1991 neutron flux from the Sun at $0.3 \mathrm{AU}$ that includes the effect of decay. We assumed that the neutron spectrum is the power law above $20 \mathrm{MeV}$ described by Debrunner et al., but at lower energies (not measured) it rolls over into an exponential spectrum $\left(E_{0}=5\right.$ $\mathrm{MeV}$ ) that would be representative of an nuclear evaporation spectrum. In Fig. 13 is the number of neutrons that would interact in different energy bins as a result of this flux at $0.3 \mathrm{AU}$. The instrument under these conditions would detect about 500 elastic n-p double-scatter events above $20 \mathrm{MeV}$, but it would also detect about 6200 elastic n-p single-scatter events above $10 \mathrm{MeV}$. The double-scatter events with the higher threshold of $20 \mathrm{MeV}$ would be used to image the Sun for the most sensitive detection as well as for spectroscopy. The elastic n-p single-scatter events can be used for spectroscopy, if the $\mathrm{S} / \mathrm{N}$ ratio is high enough for a background subtraction. Compared to that at $1 \mathrm{AU}$ the $\mathrm{S} / \mathrm{N}$ ratio would be much greater at $0.3 \mathrm{AU}$ for three reasons: (1) the $r^{2}$ effect, (2) much fewer losses due to decay at the low energies and (3) the event is compressed in time because of much smaller velocity dispersion at this location.

The incident spectrum at $1 \mathrm{AU}$ is shown in Fig. 14. Compared to that at 0.3 $\mathrm{AU}$, the flux at $10 \mathrm{MeV}$ is greatly reduced due to decay. The resulting counts for different modes is shown in Fig. 15. Here, the instrument would detect only 9 elastic n-p double-scatter events above $20 \mathrm{MeV}$, and only about 50 elastic n-p scatter above $10 \mathrm{MeV}$. Clearly, a larger instrument would be desirable at $1 \mathrm{AU}$. Increasing the linear dimension from 10 to 20 $\mathrm{cm}$ would increase the effective area more than simply the frontal area. For the same event, using an older and simpler simulation model, we predict that a $22 \mathrm{~cm}$ cube SONTRAC would detect 270 solar neutrons in the energy range 20-100 $\mathrm{MeV}$ (O'Gorman, private comm.). (These calculations agree with the newer calculations when using a $10 \mathrm{~cm}$ instrument.) This response is superior to that of COMPTEL on the Compton Gamma Ray Observatory

(CGRO) for detecting and measuring neutrons, and in a much more compact and lightweight instrument with higher count-rate capabilities. With this response the $\mathrm{S} / \mathrm{N}$ ratio of the $22 \mathrm{~cm}$ instrument would still be high. Assuming an isotropic distribution of the background neutrons, such as measured by COMPTEL ${ }^{16}$, we calculate that, for the same event, SONTRAC in a low Earth orbit would "detect" 40 background neutrons from the solar direction. This results in a detection significance of $40 \sigma_{-}$as compared to a $5 \sigma$ detection for COMPTEL ${ }^{17}$.

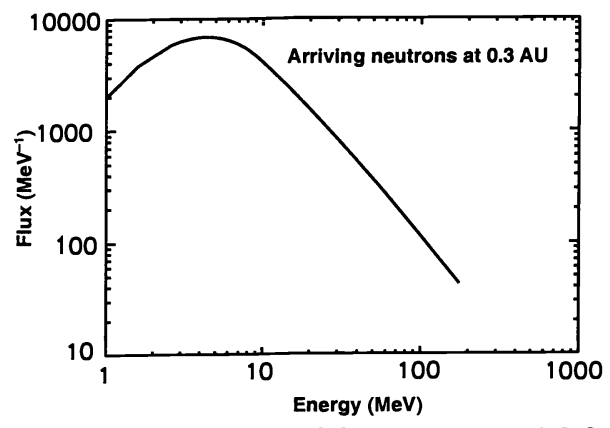

Fig. 12 Spectrum of arriving neutrons at 0.3 AU.
The typical energy resolution is on the order of $10 \%$ or better for the majority of neutron events. Angular resolution is largely determined by the pitch of the fibers, i.e., the uncertainty in the end points of the particle tracks. With a $300 \mu \mathrm{m}$ pitch and a $45^{\circ}$ scatter, the angular resolution ranges from $23^{\circ}$ at $20 \mathrm{MeV}$ to $\sim 5^{\circ}$ at $50 \mathrm{MeV}$ to $0.7^{\circ}$ at 200 $\mathrm{MeV}$.

The minimum background that the tracker must always endure will be primarily from cosmic-ray protons (and muons if flown as a balloon payload). The veto system identifies charged particles that can be rejected. Cosmic-ray protons can also be identified as having tracks that begin at the edge of the detector. Furthermore, minimum-ionizing tracks are distinguishable from heavily ionizing neutron-recoil protons

in our energy range by the lack of a Bragg peak. In short, high-quality tracks can be separated from cosmic-ray proton contamination. 
The discrimination between neutron and $\gamma_{-}$ray events in the block should approach $100 \%$. Compton scattering and pair production produce minimum ionizing tracks with heavy scattering at low energy. Bias levels or thresholds can be set to identify most of these events.

\section{AN IMPROVED SONTRAC DETECTOR DESIGN}

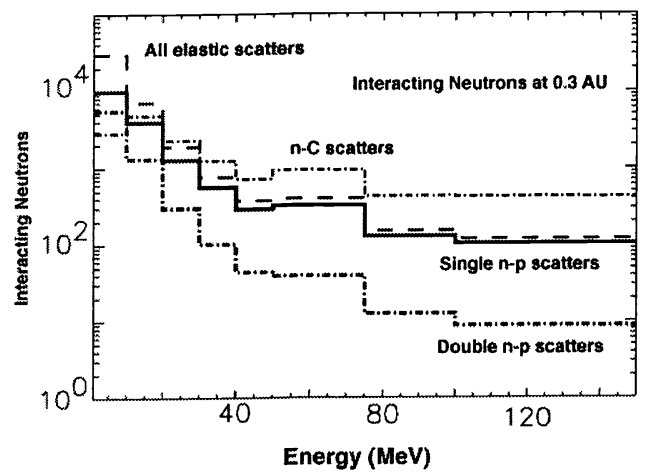

Fig. 13 Binned interacting neutrons at 0.3 in a $100 \mathrm{~cm}^{2}$ SONTRAC.

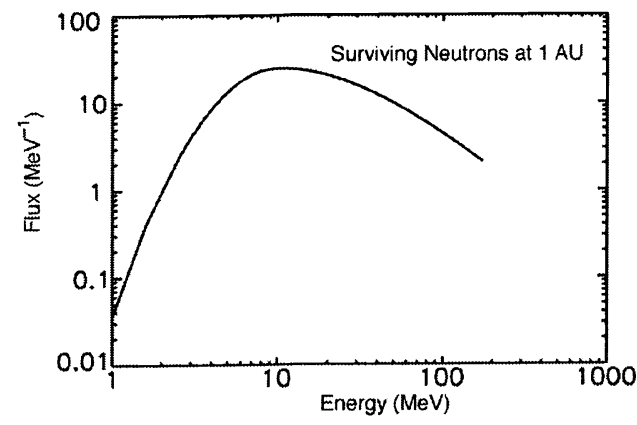

Fig. 14. Spectrum of neutrons that survive to $1 \mathrm{AU}$. lengths at lower energies. Our fiber alignment goal for future SONTRAC bundles is one fiber pitch, $\pm 300 \mu \mathrm{m}$. This will ensure that the energy and direction of recoil protons at threshold (10-20 MeV) are accurately measured. Instruments

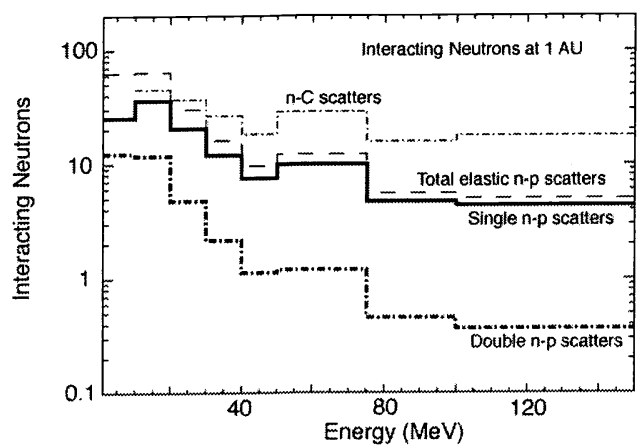

Fig. 15. Binned neutrons Interacting in a 100 $\mathrm{cm}^{2}$ SONTRAC at $1 \mathrm{AU}$

\subsection{Deficiencies in Current Detector Design}

Readout: The II-CCD readout approach employed with the SM was the most practical approach for reading the small fibers, demonstrating proof-of-principle and performing initial calibrations. This approach, however, has significant drawbacks (in two categories listed below) when applied to space- or balloon-based instrumentation.

Data Volume and Rate: The II-CCD readout scheme generates images that represent $\sim 1000 \times$ more data per event than necessary. Most fibers ( $>10^{5}$ in a $1000 \mathrm{~cm}^{3}$ bundle) provide no signal but must be read out. The fine pixel size (typically $5-10 \mu \mathrm{m}$ ) of the CCD implies that the bundle is highly over sampled, with $\sim 20 \mathrm{CCD}$ pixels covering a given fiber. Commercial CCD technologies are typically

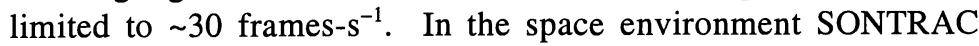
would be required to handle event rates up to $1000 \mathrm{~s}^{-1}$.

Instrument Mass and Volume: The readout hardware has considerably more volume and mass than the fiber detector bundle (See Fig. 7). Much of this is related to the hardware that minifies the image.

Triggering: PMTs are necessary for event triggering.

Fiber Bundle: The first prototype and SM fiber bundles for SONTRAC contain some misaligned fibers. While fiber positions in our prototype and SM bundles all deviate less than $\pm 1 \mathrm{~mm}$ from a rectilinear grid, such errors, if not corrected by calibration, result in degraded energy and angular resolution, particularly for short track employing scintillating plastic fibers, such as CRIS on the ACE mission ${ }^{18}$, have a successful history in space applications. The current SONTRAC scintillating plastic-fiber bundles, however, are prototypes assembled for scientific demonstration in laboratory conditions and are not designed and manufactured to survive a broad temperature range or vacuum exposure. Further engineering study with attention to the interfiber extramural absorber and adhesives is needed to establish procedures and processes for building precisely aligned and rugged scintillating-fiber bundles for SONTRAC.

\subsection{Image Projections}

Our effort uses two orthogonal 2-d (CCD) images as the basis for track reconstruction. Although effective, this track-imaging scheme can be simplified. We are studying a technique that uses three 1-d 
projections instead of two 2-d projections to reduce the data volume and increase the dynamic range. Three orthogonal 1-d projections of the track, one along each axis $(x, y, z)$, provide the unique information with which to reconstruct the length and orientation of the track in three dimensions.

For images containing complex shapes and patterns, multiple 1-d projections lead to significant information loss and reconstruction ambiguity. However, SONTRAC images contain only simple straight-line particle ionization tracks. The directionality (up vs. down discrimination) is recovered by identifying the Bragg peak at the end of the proton track. Figure 16 shows a 2-d image of a $67.5 \mathrm{MeV}$ proton, obtained with the SONTRAC Science Model, and the equivalent 1-d projections (created in software). The Bragg peak is clearly visible and the orientation of the particle track is easily obtained using the lengths in the projections.
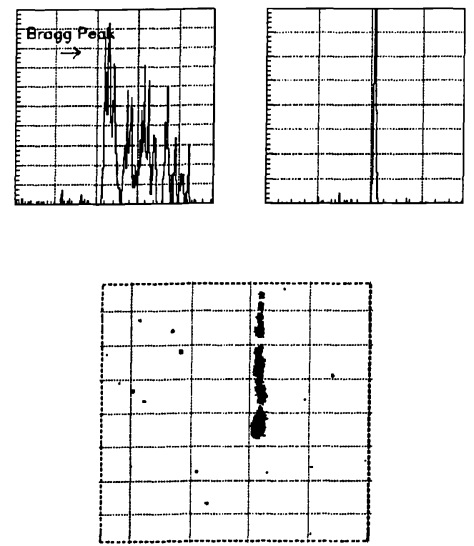

Fig. 16. 1-d projections (top) of 2-d proton track image from the SONTRAC Science Model.
Multiple recoil proton tracks, such as those expected for double np scatters could lead to event reconstruction ambiguity if the track projections overlap. Particle tracks that overlap in only one 1-d projection can be fully reconstructed without ambiguity using information from the two other projections. To evaluate the effect of multi-track overlap, neutron events, in the energy range 30-100 $\mathrm{MeV}$, were generated using the existing SONTRAC Monte Carlo. We found that $99 \%$ (95\%) of the double n-p scatters produced by 30 (100) $\mathrm{MeV}$ incident neutrons can be reconstructed without ambiguity caused by overlap. This analysis made no use of the identified Bragg peak which, when used, will further improve these efficiencies. The Bragg peak can be identified correctly with an efficiency exceeding $90 \%$.

\subsection{Strip Anode Image Tube}

Motivated by the limitations inherent in the II-CCD readout scheme, we have reviewed alternative readout devices including

CMOS imagers, intensified amorphous silicon arrays, multi-anode PMTs, avalanche photodiode arrays and image tubes with various anode configurations. Significant advantages can be obtained by a readout device with the following key properties: sensitive area matched to the fiber bundle size; spatial resolution matching the fiber size and pitch, selftriggering; fast, sparse readout; rugged; low power and compact. Also important are the advantages inherent in using multiple 1-d track projections as described above. Large-area image tubes with a strip-anode readout satisfy these requirements.

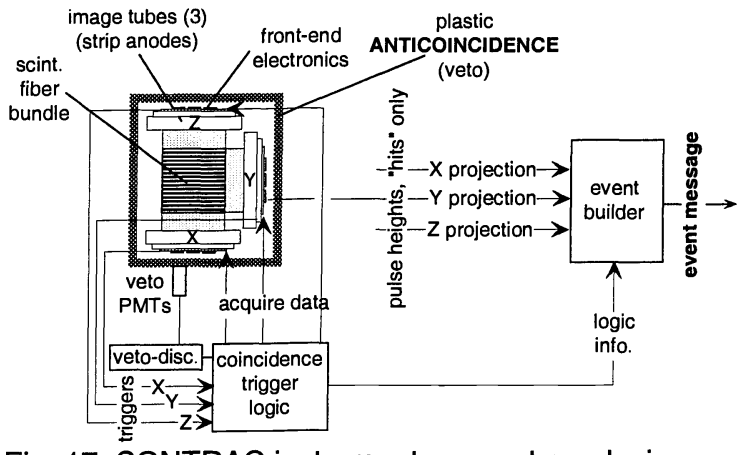

Fig. 17. SONTRAC instrument concept employing strip-anode image tube readout.
In this scheme a strip-anode image tube views a full side of the scintillating-fiber bundle. The light piped to one of the faces of the fiber bundle is detected by the image tube and is integrated along numerous parallel strips into readout electronics, yielding a 1-d projection of the light reaching that face. Figure 17 illustrates a SONTRAC detector (inside the box) using a low-profile readout device on each of three faces. Compared to the SM this results in a significant reduction of instrument volume (Fig. 7). Such a detector employing stripanode image tubes would occupy $17 \%$ of the SM volume and less than one-third the mass of one with the II-CCD/PMT readout.

Figure 18 is a cross-section view illustrating the major components of a large format, low profile $(10 \times 10 \mathrm{~cm}$ active

area) image tube. As with the image intensifiers used in the SM readout, the scintillation light is guided through a fiber optic input window and detected with a photocathode. The photoelectrons are amplified with microchannel plates in a 
sealed tube system. Unlike the image intensifier, the image tube has no phosphor to convert the amplified electron signal back into an optical image. Instead, these electrons are directly collected on parallel anode strips and measured with pulse-processing electronics. The anode strips are mutually parallel so that the readout provides a 1-d projection of the incident light distribution on that detector face.

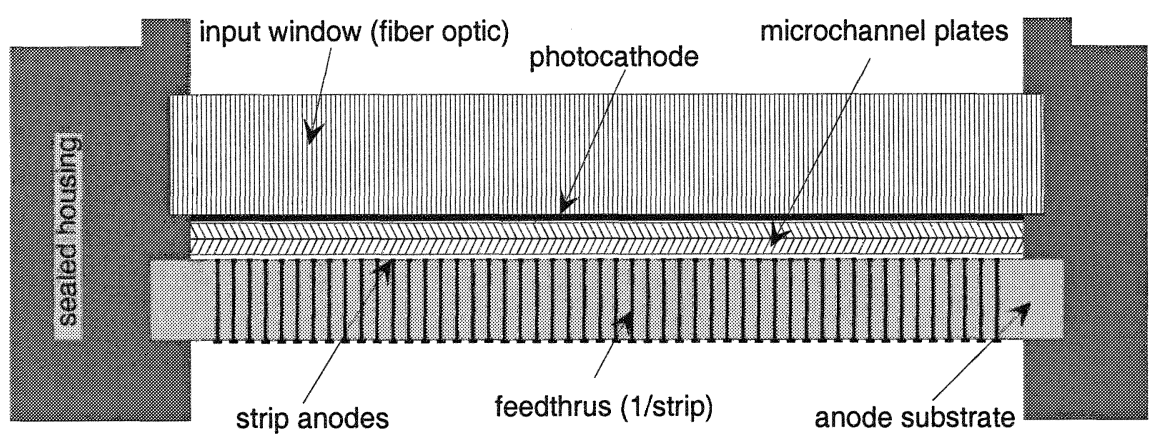

Fig. 18. Cross section of large format strip anode image tube.

Figure 19 illustrates the stripanode geometry relative to one surface of the fiber bundle. The anode surface is perpendicular to the measured fiber axis and its strips are oriented to measure one of three orthogonal projections ( $x$ in the figure). The readout scheme is illustrated for a recoil proton passing through a set of "hit" fibers. Note that only "hit" fiber channels are read out and that the resulting $1-d$

projection provides the track end points, $\mathbf{x}_{0}$ and $\mathbf{x}_{1}$, and direction.

The use of strip-anode image tube readout technology offers a number of attractive advantages for SONTRAC including: (1) significant reduction of mass and volume, (2) sufficient dynamic range (event rates $>10,000 \mathrm{~s}^{-1}$ ), (3) significant reduction of channel count and data volume, (4) complexity reduction in the optical readout chain to maintain the integrity of the signal, (5) the technology is scalable to larger applications, (6) the instrument is self-triggering, and (7) all components have a strong space heritage.

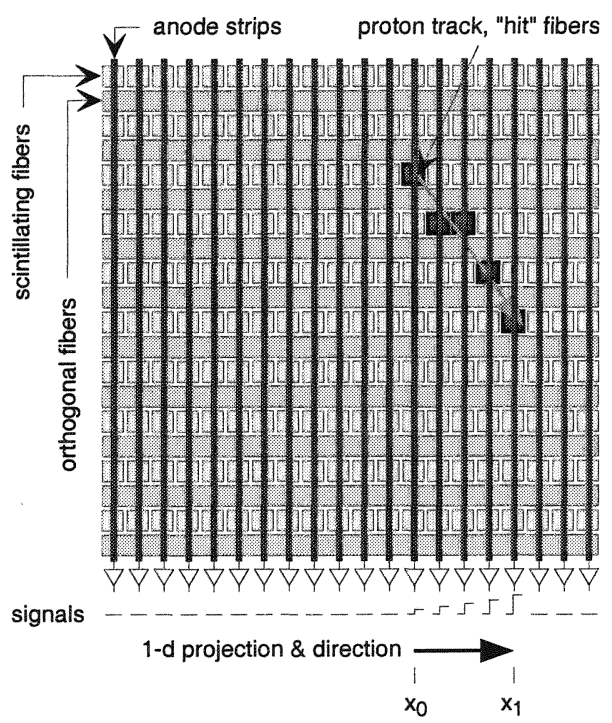

Fig. 19. Fiber bundle and anode strip geometry. Proton track response is also illustrated.

\section{CONCLUSIONS}

The SONTRAC design and concept holds out promise for the next generation of solar neutron measurements during the next solar maximum period around 2010, whether these measurements are performed from a balloon or a spacecraft near Earth or in the inner heliosphere. For an instrument positioned at $0.3 \mathrm{AU}$ (e.g., Solar Sentinel) with $1 \mathrm{~kg}$ of active mass, the neutron sensitivity far exceeds that of any instrument flown to date. The energy range will also be expanded due to the reduction in the decay losses. At Earth, a small-satellite instrument with $8 \mathrm{~kg}$ of active mass, would at least duplicate the power of COMPTEL in measuring neutrons, but with fewer mass restrictions on a balloon platform, the active mass could be made considerably larger by "tiling" SONTRAC modules into a large package. Advancements in scintillating-fiber technology, optoelectronic readouts and on-board processing makes this possible.

\section{REFERENCES}

1. Hudson, H. and J. Ryan, "High-Energy Particles in Solar Flares," Annual Review Astronomy and Astrophysics, 33, pp. 239-82, 1995. 
2. Vestrand, W.T. and J.A. Miller, "Particle Acceleration During Solar Flares," The Many Faces of the Sun: A Summary of the Results from NASA's Solar Maximum Mission, Eds. K.T. Strong, et al., pp. 231-272, Springer-Verlag, New York, 1999.

3. Ryan, J.M., J.A. Lockwood, and H. Debrunner, "Solar Energetic Particles," Space Science Reviews, 93, pp. 35-53, 2000.

4. Lockwood, J.A., H. Debrunner, and J.M. Ryan, "The Relationship between Solar Flare Gamma-Ray Emission and Neutron Production," Solar Physics, 173, pp. 151-176, 1997.

5. Murphy, R.J., C.D. Dermer, and R. Ramaty, "High-Energy Processes in Solar Flares," The Astrophysical Journal (Supplement Series), 63, pp. 721-748, 1987.

6. Share, G.H. and R.J. Murphy, "Gamma-Ray Measurements of Flare-to-Flare Variations in Ambient Solar Abundances," The Astrophysical Journal, 452, pp. 933-943, 1995.

7. Ryan, J., et al. "Neutron and Gamma Ray Measurements of the Solar Flare of 1991 June 9," High-Energy Solar Phenomena-A New Era of Spacecraft Measurements, 294, pp. 89-93, AIP, New York, 1994.

8. Schneid, E.J., et al. "EGRET Observations of Extended High Energy Emissions from the Nuclear Line Flares of June 1991," High-Energy Solar Phenomena-A New Era of Spacecraft Measurements, 294, pp. 94-99, AIP, New York, 1994.

9. Ryan, J.M., "Long Duration Solar Gamma-Ray Flares," Space Science Reviews, 93, pp. 581-610, 2000.

10. Wunderer, C.B., et al. "SONTRAC-A Low Background, Large Area Solar Neutron Spectrometer," Conference on the High Energy Radiation Background in Space, 97TH8346, pp. 73-76, IEEE, Snowmass, 1997.

11. Ryan, J.M., et al. "A Prototype for SONTRAC, a Scintillating Plastic Fiber Detector for Solar Neutron Spectroscopy,” SPIE, 3114, pp. 514-525, San Diego, 1997.

12. Ryan, J.M., et al., "A scintillating plastic fiber tracking detector for neutron and proton imaging and spectroscopy," Nuclear Instruments \& Methods in Physics Research, 422, pp. 49-53, 1999.

13. Ryan, J.M., et al. "Recent progress in the development of a solar neutron tracking device (SONTRAC)," Hard XRay, Gamma-Ray, and Neutron Detector Physics, 3768, pp. 496-502, SPIE, Denver, 1999.

14. Desorgher, L., et al. "Geant Monte Carlo Simulation of the SONTRAC detector," International Cosmic Ray Conference. SH, pp. 3065-3068, Copernicus Systems and Technology GmbH, Berlin, Germany, Hamburg, 2001.

15. Debrunner, H., et al. "Neutrons from the 15 June 1991 solar flare," 23rd International Cosmic Ray Conference. 3, pp. 115-118, Calgary, 1993.

16. Morris, D.J., et al., "Neutron measurements in near-Earth orbit with COMPTEL," Journal of Geophysical Research, 100, pp. 12,243-12,249, 1995.

17. Rank, G., et al. "Extended $\gamma$-Ray Emission in Solar Flares," High Energy Solar Physics, 374, pp. 219-236, AIP, New York, 1996.

18. Stone, E.C., et al., "The Cosmic-Ray Isotope Spectrometer for the Advanced Composition Explorer," Space Science Reviews, 86, pp. 285-356, 1998. 